\title{
The Health Effects of Antenatal Multiple Micronutrient Supplementation in Nepalese Children
}

\author{
Delan Devakumar $^{1^{*}}$, Jonathan Wells ${ }^{1}$, Shiva Chaube ${ }^{2}$, Naomi Saville ${ }^{1}$, \\ Dharma Manandhar ${ }^{2}$, Anthony Costello ${ }^{1}$, Jonathan Ayres ${ }^{3}$, \\ Janet Stocks ${ }^{1,4}$ and David Osrin ${ }^{1}$ \\ ${ }^{1}$ University College London, London, UK. \\ ${ }^{2}$ Mother and Infant Research Activities, Kathmandu, Nepal. \\ ${ }^{3}$ University of Birmingham, Birmingham, UK. \\ ${ }^{4}$ Great Ormond St Hospital for Children NHS Trust Foundation, London, UK.
}

Article Information

DOI: 10.9734/EJNFS/2015/21337

Special Editors:

Lucie Bohac, Micronutrient Forum Secretariat, Canada. Klaus Kraemer, Director, Sight and Life, Basel, Switzerland.

Chief Editor

Prof. Hans Verhagen, Senior Scientific Advisor 'Nutrition and Food Safety', National Institute for Public Health and the Environment (RIVM), P.O.Box 1, 3720 BA, Bilthoven, The Netherlands.

\begin{abstract}
Objectives: In low- and middle-income countries, prenatal multiple micronutrient supplementation has been shown in meta-analyses to increase birthweight and reduce the proportion of babies born small-for-gestational-age, but the longer term effects are unclear. We examined phenotypic and health differences between children born to mothers in a randomized controlled trial of prenatal multiple micronutrient (UNIMAPP) supplementation compared to iron and folate.

Methods: The study was conducted in Dhanusha district, Nepal. We measured anthropometry, body composition using bioelectrical impedance (with population-specific isotope calibration), blood pressure, kidney dimensions by ultrasound, and spirometry. Health outcomes were recorded in questionnaires. Personal estimates of air pollution exposure (particles of less than 4 micrometers in size) were produced by microenvironment sampling.

Results: We saw 841 children ( $80 \%$ of total), at a mean age of 8.5 years. The unadjusted mean $(95 \% \mathrm{Cl})$ difference (intervention minus control) in weight-for-age was $0.05 \mathrm{z}$-scores $(-0.09,0.19)$, in height-for-age 0.02 z-scores $(-0.10,0.15)$ and in BMl-for-age 0.04 z-scores $(-0.09,0.18)$. Similar results were obtained from multi-variable regression analysis. No difference was found in body
\end{abstract}

Correspondence: Lucie Bohac, Coordinator, Micronutrient Forum Secretariat (email: Ibohac@micronutrient.org) Note: This abstract was presented at "The Micronutrient Forum Global Conference - Bridging Discovery and Delivery", 2-6 June 2014, Addis Ababa, Ethiopia. 
composition, kidney size, blood pressure or lung function. In addition there were no differences in health outcomes between allocation groups.

Conclusions: Differences that were previously seen in phenotype and blood pressure at birth and at two years in children born to mothers who received antenatal UNIMMAP supplements were not apparent at 8.5 years of age. While not ruling out physiological differences and health benefits in adulthood, our findings do not suggest sustained effects of antenatal micronutrient supplementation.

(c) 2015 Devakumar et al.; This is an Open Access article distributed under the terms of the Creative Commons Attribution License (http://creativecommons.org/licenses/by/4.0), which permits unrestricted use, distribution, and reproduction in any medium, provided the original work is properly cited. 